\title{
Quantitative Analysis of the Macular and Peripapillary Capillary Network with Optical Coherence Tomography Angiography in Chinese Adolescents: The Tuyou County Pediatric Eye (TYPE) Study
}

This article was published in the following Dove Press journal:

International Journal of General Medicine

\author{
Yajun Yang $\mathbb{D}^{1, *}$ \\ Guisen Zhang $\mathbb{D}^{2, *}$ \\ Song Zhang ${ }^{3, *}$ \\ Xiaoyan Bian' \\ Lao $\mathrm{Qi}^{2}$ \\ Shixuan Guo ${ }^{2}$ \\ Bozhou Zhang' \\ Lei Liu (1D ${ }^{4}$ \\ 'Cataract Department, Baotou Chaoju \\ Eye Hospital, Baotou, 014060, People's \\ Republic of China; ${ }^{2}$ Retina Department \\ Hohhot Chaoju Eye Hospital, Hohhot, \\ 0I0050, People's Republic of China; \\ ${ }^{3}$ Cataract Department, China Medical \\ University, Shenyang, II0122, People's \\ Republic of China; ${ }^{4}$ Department of \\ Ophthalmology, The First Affiliated \\ Hospital of China Medical University, \\ Shenyang, II000I, People's Republic of \\ China
}

*These authors contributed equally to this work
Correspondence: Bozhou Zhang Baotou Chaoju Eye Hospital, Baotou, Inner Mongolia Autonomous Region, 014060, People's Republic of China Email zhangbozhou@chaojueye.cn

Lei Liu

The First Affiliated Hospital of China Medical University, Shenyang, Liaoning, I I000I, People's Republic of China Email liuleijiao@I63.com

\begin{abstract}
Aim: To use OCTA to collect normative data describing the vascular perfusion of the macula and optic disc in adolescents.

Methods: This cross-sectional, school-based study was conducted from Oct 15, 2019, to Nov 30, 2019, in Tuyou County, Baotou, China. All eligible participants underwent a comprehensive questionnaire and ocular examination. The vascular perfusion of the macula and optic disc was determined using a spectral-domain OCTA device.

Results: A total of 570 anatomically normal eyes without a history of pathologic disease from 570 adolescents (mean \pm SD age, $15.1 \pm 1.9$ years; 298 girls [52.3\%]) were enrolled. In the macula, the mean \pm SD perifoveal perfusion density (PD) of the superficial capillary plexus (SCP) and deep capillary plexus (DCP) was $44.2 \% \pm 4.37 \%$ and $50.74 \% \pm 3.98 \%$, respectively. The mean $\pm \mathrm{SD}$ foveal avascular zone (FAZ) was $0.32 \mathrm{~mm}^{2} \pm 0.11 \mathrm{~mm}^{2}$. The mean \pm SD peripapillary vessel density (PVD) was $54.98 \% \pm 3.53 \%$. The inferior hemiretinal SCP-PD, DCP-PD, FAZ, and PVD was larger in girls than boys $(P=0.006, P=$ $0.001, P<0.001$, and $P=0.006$, respectively) Multiple regression analysis confirmed that sex independently affected the DCP-PD, FAZ, and PVD $(P<0.001, P<0.001$, and $P=$ 0.029 , respectively) and that axial length (AL) independently affected the FAZ area and PVD ( $P=0.004$ and $P<0.001$, respectively).

Conclusion: Quantitative studies of the perifoveal vasculature in adolescents should consider the patient's sex and AL. Our findings may provide useful information for the understanding and the management of retinal perfusion in Chinese adolescents.
\end{abstract}

Keywords: macula, optic disc, optical coherence tomography angiography, retinal perfusion

\section{Introduction}

Puberty represents a critical period of organ growth in adolescents, including that of ocular development. ${ }^{1}$ Understanding healthy adolescent microvascular development and any deviation from normal could indicate the development of disease. Previously, fluorescein angiography (FA) was used to evaluate pediatric vascular disorders, although this technique is invasive and patients may experience some adverse effects. $^{2}$ Recently, optical coherence tomography angiography (OCTA), a noninvasive study that does not require the use of contrast material, has greatly advanced physicians' ability to determine diseases affecting retinal vasculature such as diabetic retinopathy (DR), retinopathy of prematurity (ROP), neovascular age-related 
macular degeneration (nAMD), familial exudative vitreoretinopathy (FEVR), Coats' disease and disorders of the optic nerve. ${ }^{3-8}$ Furthermore, the use of OCTA in adolescents to examine their retinal microvasculature may provide insight into healthy microvascular development during the pubescent period.

In addition, OCTA has the advantage of better visualization of the superficial and deep vascular layers of the macula, including the superficial capillary plexus (SCP) and deep capillary plexus (DCP). ${ }^{8,9}$ Further, OCTA images can be used to quantitatively analyze the foveal avascular zone (FAZ), which is a capillary-free region in the retina. ${ }^{10}$ With OCTA, it is possible to quantitatively evaluate blood flow at the optic disc, allowing for the measurement of peripapillary vessel density (PVD).

Most studies using OCTA to quantify healthy retinal perfusion have been performed in adults; however, to our knowledge, few clinical studies have included an analysis of children. Previous studies have been limited by their hospital-based designs and small sample size of the included participants. ${ }^{10-14}$ In addition, the results of one clinical study using a small sample size revealed that several variables, such as age and sex, could influence the density of the macular microvasculature; ${ }^{15}$ how these factors manifest themselves within the vascular perfusion of the optic disc in adolescents is still virtually unknown.

To address these issues, we conducted a school-based cross-sectional study to measure macular and optic disc vasculature in healthy adolescents using OCTA.

\section{Methods}

The school-based, cross-sectional Tuyou Pediatric Eye (TYPE) Study was carried out from Oct 15, 2019, to Nov 30, 2019, in the Tuyou County of Baotou in the Inner Mongolia Autonomous Region, China. The TYPE study was conducted to compare variations in the prevalence and associated factors of refractive error, ocular surface, and retinal abnormalities among different ethnic groups of adolescents aged 11 to 18 years. Briefly, two middle (junior and senior) schools were selected by a multistage cluster sampling strategy. First, given the heavily unbalanced development in China, Tuyou County of Baotou City in northern China represents an administrative division and was selected from the Inner Mongolia Autonomous Region, China. Second, one senior middle school and one junior middle school that represent the average education level were selected from the suburban area of Tuyou County, respectively. Finally, two classes were randomly selected from each grade at each school. All 687 students from those classes were recruited to the survey; 20 students did not attend the investigation, leaving 667 (response rate, 97.1\%) students to complete the questionnaires and all ocular examinations. We included the participants with a spherical equivalent (SE) ranging from -6.0 to +6.0 diopters, a best-corrected visual acuity greater than or equal to 1.0 or 5.0, and those without any evidence of strabismus or intraocular disease as determined by an ophthalmologist. Participants were excluded from the current study if they had any systemic medical condition that could affect the retinal vasculature, or had prior trauma or intraocular surgeries. Data from the right eyes of all subjects were considered for the current analysis. This study complied with the declaration of Helsinki and was approved by the institutional review board of Baotou Chaoju Eye Hospital (No. CHAOJU-BT-2020003), and all participants or their parents gave written informed consent. This study followed the Strengthening the Reporting of Observational Studies in Epidemiology (STROBE) guidelines (https://www.strobestatement.org).

\section{Demographic Characteristics}

Demographic information, including age, sex, family socioeconomic status, and lifestyle, such as sleep duration per day, were collected via questionnaires. Sleep duration per day was ascertained using the following question, "During your longest or nocturnal sleep period, what time do you normally go to bed and wake up?" (Time was recorded in minute format).

\section{Body Check}

Each participant underwent a medical examination including weight, height, systolic blood pressure (SBP), and diastolic blood pressure (DBP). Body mass index (BMI) was calculated by dividing body weight in kilograms by the square of the height in meters.

\section{Ocular Examination}

Further, a comprehensive ophthalmologic examination was conducted including best-corrected visual acuity (BCVA) testing, ocular biometry with the IOL Master 500 (Zeiss Meditec, Inc., Dublin, CA), fundus examination using nonmydriatic cameras TRC-NW8 (Topcon Corporation, Tokyo, Japan), and spectral-domain OCTA (AngioVue; Optovue, Fremont, CA) assessment. SE was calculated as the sphere plus half the cylindrical power. 
Split-spectrum amplitude-decorrelation angiography algorithm (SSADA) on the AngioVue OCT-A system version 2017.1.0.15 (OptovueRTVue XR Avanti, Optovue Inc., Freemont, California, USA) was used to obtain OCTA images. Volume scans approximately $6 \times 6 \mathrm{~mm}$ in size were centered onto the fovea, and a $4.5-\times 4.5-\mathrm{mm}$ scan field was centered on the optic disc in both eyes of every patient, respectively. Images with quality less than 7, vitreous floaters, artifacts, or segmentation failures were excluded from the analysis. The OCT images were taken in school and performed in both eyes.

The densities of the SCP and DCP were measured using fractal analysis by box-counting (Dbox). We identify projection artifact by evaluating the correlation of each line with adjacent rows or columns. Motion abnormalities were performed by tracking-assisted scanning integrated with motion correction technology (MCT). A rectangle scan of $4.5 \times 4.5 \mathrm{~mm}$ centered on the optic nerve head $(\mathrm{ONH})$ was obtained using Angio-Disc mode. The software automatically fits a $2.0 \mathrm{~mm}$ diameter circle, centered on $\mathrm{ONH}$, and defines a circle $2.0 \mathrm{~mm}$ wide.

\section{Definition}

Peripapillary vessel density is measured in a $0.75-\mathrm{mm}$ wide elliptical annulus extending outward from the optic disc boundary. ${ }^{16}$ The parafovea was defined as the area within the annulus located between $1 \mathrm{~mm}$ and $2.5 \mathrm{~mm}$ from the central fovea. ${ }^{17}$ The SCP and DCP measurements were performed using the inbuilt software algorithm. The SCP was set at $3 \mu \mathrm{m}$ below the internal limiting membrane of the retina and the outer boundary at $15 \mu \mathrm{m}$ beneath the inner plexiform layer (IPL), whereas the DCP top boundary was set at $15 \mu \mathrm{m}$ beneath the IPL and the bottom margin at $71 \mu \mathrm{m}$ under the IPL. Vessel density was defined as the percentage of area occupied by vessel lumens after the binary reconstruction of images. In addition, vessel density was calculated for different retinal sectors (superior, nasal, inferior, and temporal), which were automatically determined from the OCTA. The FAZ was measured by the polygon selection tool of the ImageJ software (National Institutes of Health, Bethesda, MD; available at http://rsb.info.nih.gov/ij/index.html).${ }^{10}$ A qualitative evaluation of the OCTA images was also performed by two double-masked ophthalmologists (X.Y.B. and Y.J.Y.). In unclear situations, a senior ophthalmologist (G.S.Z.) reassessed the images to arrive at a final consensus.

\section{Statistical Analysis}

Statistical analysis was performed using the SPSS software, version 23.0 (IBM Corp, USA). Data obtained from each group were compared using one-way analysis of variance (ANOVA). Univariate regression analysis was used to determine relationships between systematic variables (eg, age, sex, SBP, DBP, and BMI) and OCTA parameters. Multivariate regression analysis coefficients $(\beta)$ were used to assess age, sex, and interaction in models for macular and optic disc vessel density. $P$ values were two sided, and a value of $P<0.05$ was considered statistically significant. All statistical analyses were performed from Month Day, Year, to Month Day, Year.

\section{Results}

Altogether, 667 individuals were enrolled in the current study. After screening, 97 were excluded from vessel density analysis because of artifacts affecting image quality, leaving a total of 570 anatomically normal eyes without a history of pathologic disease from 570 adolescents (mean \pm SD age, $15.1 \pm 1.9$ years; 298 girls [52.3\%]). The mean $\pm \mathrm{SD}$ axial length (AL) was $23.96 \pm 1.31 \mathrm{~mm}$. There was no significant difference in age, sex, and AL between the included and the excluded eyes $(P=0.42, P=0.25$, and $P=0.36$, respectively). The mean $\pm \mathrm{SD}$ of the SCPPD, DCP-PD, and FAZ were $44.2 \% \pm 4.37 \%, 50.74 \% \pm$ $3.98 \%$, and $0.32 \mathrm{~mm}^{2} \pm 0.11 \mathrm{~mm}^{2}$, respectively. For the optic disc measurements, the mean \pm SD PVD value was $54.98 \% \pm 3.53 \%$.

\section{Outcomes Measurements Stratified by Age}

There were significant differences in weight $(P<0.001)$, BMI $(P=0.019)$, sleep duration per day $(P<0.001)$, and AL $(P=0.004)$ according to age. However, there were no significant differences between the age groups regarding SCP-PD, DCP-PD, FAZ, and PVD, respectively (all $P>$ 0.05) (Table 1).

\section{Outcome Measurements Stratified by Sex}

The inferior hemiretinal including inferior nasal and inferior temporal SCP-PD, DCP-PD, FAZ, and PVD was larger in girls than in boys $(P<0.001, P=0.006, P=0.001, P<$ 0.001 , and $P=0.006$, respectively), whereas $\mathrm{AL}$ was shorter in girls than in boys $(P=0.003)$. However, age, BMI, SBP, DBP, sleep duration per day, SCP-PD, and the temporal hemiretinal PVD values did not differ 


\begin{tabular}{|c|c|c|c|c|c|}
\hline$a$ & 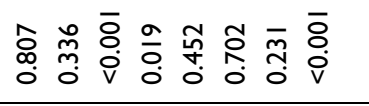 & $\begin{array}{l}\bar{n} \\
\text { Oั } \\
0\end{array}$ & 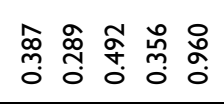 & 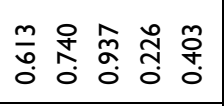 & 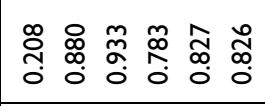 \\
\hline 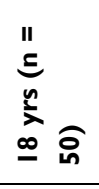 & 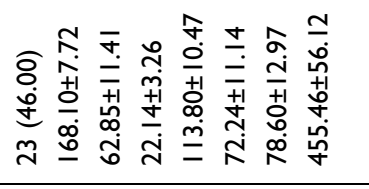 & 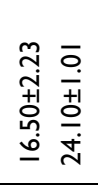 & 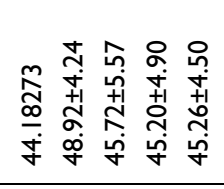 & 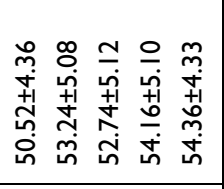 & 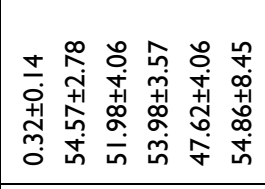 \\
\hline 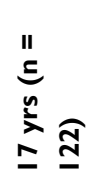 & 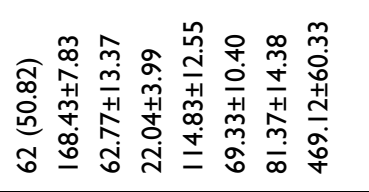 & 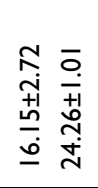 & 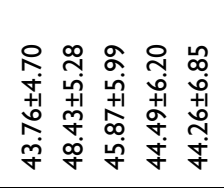 & 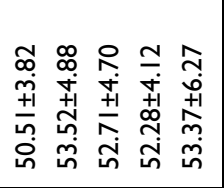 & 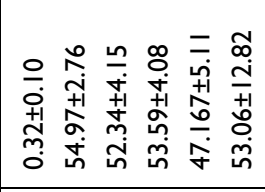 \\
\hline 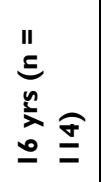 & 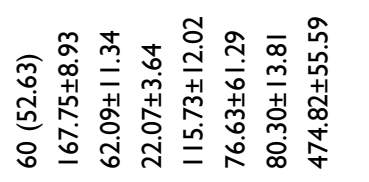 & 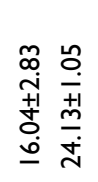 & 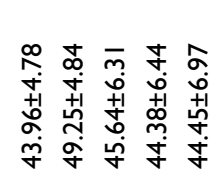 & 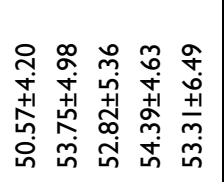 & 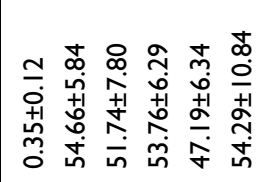 \\
\hline 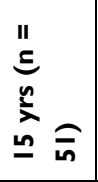 & 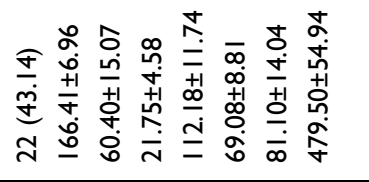 & 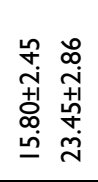 & 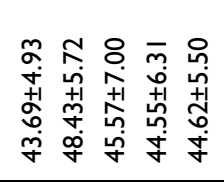 & 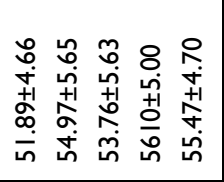 & 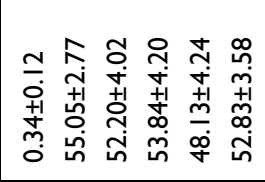 \\
\hline 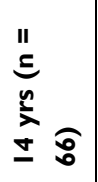 & 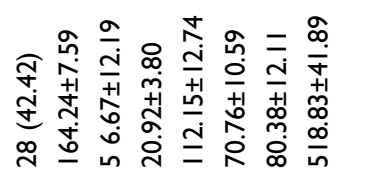 & 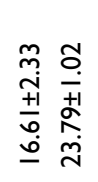 & 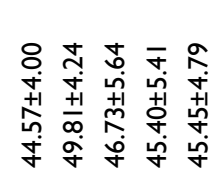 & 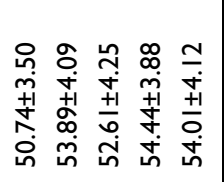 & 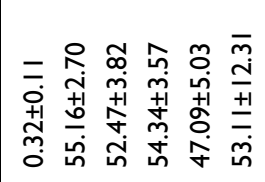 \\
\hline 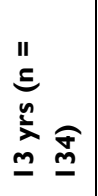 & 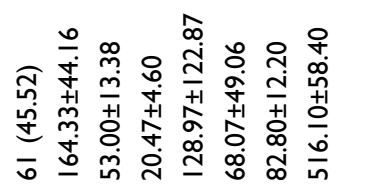 & 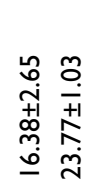 & 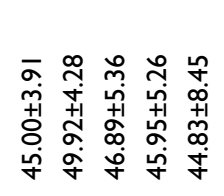 & 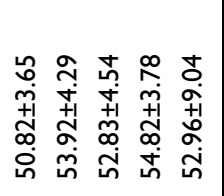 & 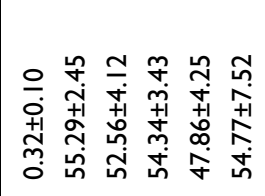 \\
\hline 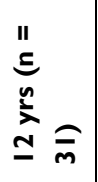 & 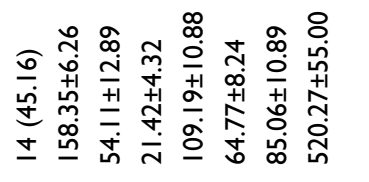 & 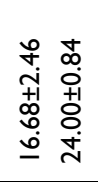 & 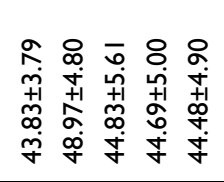 & 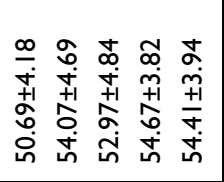 & 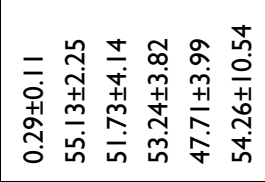 \\
\hline 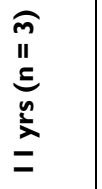 & 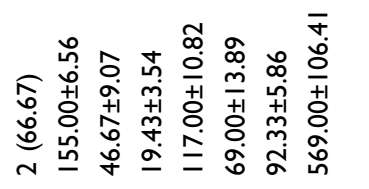 & 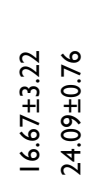 & 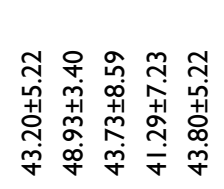 & 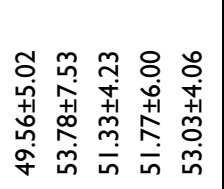 & 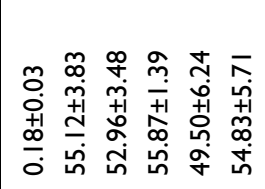 \\
\hline 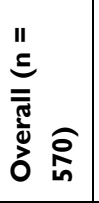 & 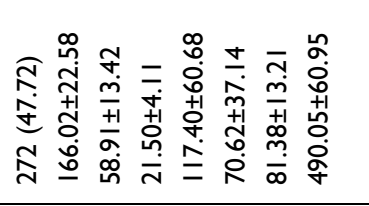 & 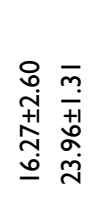 & 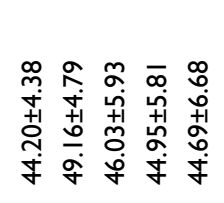 & 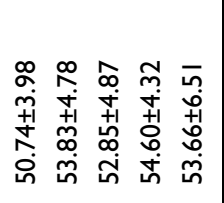 & 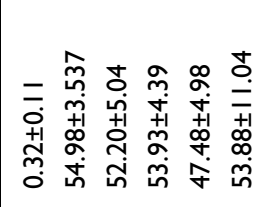 \\
\hline & 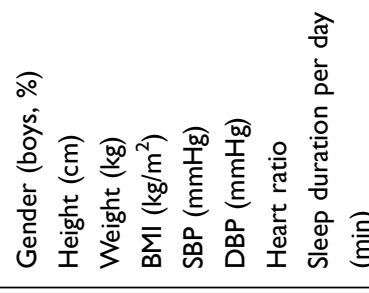 & 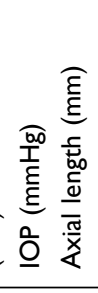 & 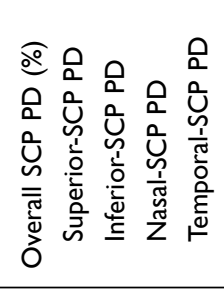 & 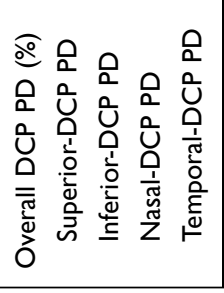 & 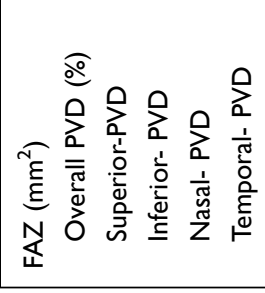 \\
\hline
\end{tabular}


Table 2 Adolescent Characteristics by Sex

\begin{tabular}{|c|c|c|c|c|}
\hline & Overall $(n=570)$ & Boys $(n=272)$ & Girls $(n=298)$ & $P$ \\
\hline Age (yrs) & $15.12 \pm 1.86$ & $15.19 \pm 1.86$ & $15.05 \pm \mid .87$ & 0.355 \\
\hline Height (cm) & $166.02 \pm 22.58$ & $170.05 \pm 8.20$ & $162.35 \pm 29.79$ & $<0.001$ \\
\hline Weight (kg) & $58.91 \pm 13.42$ & $62.21 \pm 13.99$ & $55.89 \pm 12.14$ & $<0.001$ \\
\hline BMI $\left(\mathrm{kg} / \mathrm{m}^{2}\right)$ & $21.50 \pm 4.11$ & $21.40 \pm 4.04$ & $21.58 \pm 4.18$ & 0.610 \\
\hline $\mathrm{SBP}(\mathrm{mmHg})$ & $117.40 \pm 60.68$ & $122.57 \pm 72.72$ & $112.68 \pm 46.72$ & 0.052 \\
\hline $\mathrm{DBP}(\mathrm{mmHg})$ & $70.62 \pm 36.14$ & $69.76 \pm 35.06$ & $7 I .4 I \pm 38.99$ & 0.596 \\
\hline Heart ratio & $81.38 \pm 13.20$ & $79.04 \pm|4.2|$ & $83.52 \pm 11.84$ & $<0.001$ \\
\hline Sleep duration per day (min) & $490.05 \pm 60.95$ & $492.11 \pm 64.72$ & $488.16 \pm 57.34$ & 0.440 \\
\hline IOP $(\mathrm{mmHg})$ & $16.27 \pm 2.60$ & $|6.3| \pm 2.60$ & $|6.24 \pm 2.6|$ & 0.758 \\
\hline Axial length (mm) & $23.96 \pm 1.31$ & $24.13 \pm 1.55$ & $23.81 \pm 1.02$ & 0.003 \\
\hline Overall SCP PD (\%) & $44.20 \pm 4.38$ & $43.87 \pm 4.17$ & $44.50 \pm 4.54$ & 0.087 \\
\hline Superior-SCP PD & $49.16 \pm 4.79$ & $48.84 \pm 4.64$ & $49.45 \pm 4.92$ & 0.132 \\
\hline Inferior-SCP PD & $46.03 \pm 5.93$ & $45.30 \pm 5.84$ & $46.69 \pm 5.93$ & 0.006 \\
\hline Nasal-SCP PD & $44.95 \pm 5.81$ & $44.8 I \pm 5.33$ & $45.09 \pm 6.30$ & 0.563 \\
\hline Temporal-SCP PD & $44.69 \pm 6.68$ & $44.40 \pm 6.93$ & $44.95 \pm 6.44$ & 0.333 \\
\hline Overall DCP PD (\%) & $50.74 \pm 3.98$ & $50.17 \pm 4.25$ & $51.27 \pm 3.65$ & 0.001 \\
\hline Superior-DCP PD & $53.83 \pm 4.78$ & $53.12 \pm 5.10$ & $54.49 \pm 4.36$ & 0.001 \\
\hline Inferior-DCP PD & $52.85 \pm 4.87$ & $51.86 \pm 5.08$ & $53.75 \pm 4.50$ & $<0.001$ \\
\hline Nasal-DCP PD & $54.60 \pm 4.32$ & $53.86 \pm 4.47$ & $55.28 \pm 4.06$ & $<0.001$ \\
\hline Temporal-DCP PD & $53.66 \pm 6.51$ & $52.94 \pm 7.05$ & $54.33 \pm 5.90$ & 0.011 \\
\hline FAZ $\left(\mathrm{mm}^{2}\right)$ & $0.32 \pm 0.11$ & $0.30 \pm 0.11$ & $0.34 \pm 0.11$ & $<0.001$ \\
\hline Overall PVD (\%) & $54.98 \pm 3.54$ & $54.5 I \pm 4.24$ & $55.40 \pm 2.65$ & 0.003 \\
\hline Superior-PVD & $52.20 \pm 5.04$ & $51.30 \pm 6.00$ & $53.02 \pm 3.80$ & $<0.001$ \\
\hline Inferior- PVD & $53.93 \pm 4.39$ & $53.31 \pm 4.90$ & $54.49 \pm 3.79$ & 0.001 \\
\hline Nasal- PVD & $47.48 \pm 4.98$ & $46.95 \pm 5.06$ & $47.97 \pm 4.86$ & 0.016 \\
\hline Temporal- PVD & $54.02 \pm 10.63$ & $54.16 \pm 9.43$ & $53.89 \pm 11.63$ & 0.763 \\
\hline
\end{tabular}

Note: Data are presented as mean \pm SD.

Abbreviations: BMI, body mass index; DBP, diastolic blood pressure; DCP, deep capillary plexus; FAZ, foveal avascular zone; IOP, intraocular pressure; PVD, peripapillary vessel density; SBP, systolic blood pressure; SCP, superficial capillary plexus.

significantly between boys and girls $(P>0.05)$ (Table 2$)$. The description of characteristics of the included individuals by age and sex is shown in supplementary Table 1.

\section{Association Between Systematic Factors and OCTA Measurements}

Associations between demographic, systemic, and ocular variables with log-transformed OCTA metrics by univariate and multiple regression analysis are shown in Table 3. In the multiple regression model, sex was independently associated with DCP-PD, FAZ, and PVD $(\beta=0.157, P<0.001 ; \beta=0.156, P<0.001 ;$ and $\beta=$ $0.089, P=0.029$, respectively), and AL independently affected the FAZ area and PVD $(\beta=-0.123, P=0.004$, and $\beta=-0.158, P<0.001$, respectively). Furthermore, weight was an independent determinant of SCP-PD $(\beta$ $=-0.259, P=0.023$ ).

\section{Discussion}

In this hospital-based cross-sectional study, we analyzed and described the optic disc and perifoveal microvasculature in healthy adolescents. Furthermore, we assessed the relationships between the retinal vessel density with the following demographic or systematic characteristics: age, sex, sleep duration per day, AL, intraocular pressure, SBP, DBP, height, weight, and BMI.

Previously, Chu et al examined 135 normal eyes of 89 infants and children (mean age $\pm \mathrm{SD}, 8.5 \pm 5.3$ years; range, 9 weeks-17 years) treated at an eye center using OCTA software (Heidelberg Engineering, Heidelberg, Germany), and they concluded that the mean $\pm \mathrm{SD}$ of the FAZ area was $0.35 \mathrm{~mm}^{2} \pm 0.17 \mathrm{~mm}^{2}$. Further, the FAZ area did not vary significantly among the different age categories (0-2 years, 3-6 years, 7-9 years, 10-12 years, 13-15 years, and $16-18$ years), ${ }^{18}$ which was similar to our 
Table 3 Correlation Coefficient Between Demographic, Systematic, and Ocular Parameters with OCTA Metrics

\begin{tabular}{|c|c|c|c|c|c|}
\hline & Variables & Unadjusted Coefficients ( $\beta$ ) & $\mathbf{P}$ & Adjusted Coefficients $(\beta)^{*}$ & $P$ \\
\hline \multirow[t]{14}{*}{ Overall SCP PD (\%) } & Age (yrs) & -0.070 & 0.097 & 0.000 & 0.998 \\
\hline & Gender & 0.072 & 0.087 & 0.014 & 0.774 \\
\hline & Height $(\mathrm{cm})$ & -0.048 & 0.262 & - & - \\
\hline & Weight (kg) & -0.139 & 0.001 & -0.259 & 0.023 \\
\hline & BMI $\left(\mathrm{kg} / \mathrm{m}^{2}\right)$ & 0.085 & 0.045 & 0.165 & 0.124 \\
\hline & $\mathrm{SBP}(\mathrm{mmHg})$ & 0.053 & 0.215 & - & - \\
\hline & $\mathrm{DBP}(\mathrm{mmHg})$ & -0.092 & 0.030 & -0.077 & 0.051 \\
\hline & Heart ratio & 0.066 & 0.121 & - & - \\
\hline & Sleep duration per day (mins) & -0.022 & 0.605 & - & - \\
\hline & IOP $(\mathrm{mmHg})$ & -0.062 & 0.143 & - & - \\
\hline & Axial length (mm) & -0.048 & 0.259 & - & - \\
\hline & Overall DCP PD (\%) & -0.250 & $<0.001$ & -0.249 & $<0.001$ \\
\hline & FAZ $\left(\mathrm{mm}^{2}\right)$ & -0.015 & 0.723 & - & - \\
\hline & Overall PVD & 0.266 & $<0.001$ & 0.244 & $<0.001$ \\
\hline \multirow[t]{14}{*}{ Overall DCP PD (\%) } & Age (yrs) & -0.027 & 0.521 & -0.042 & 0.308 \\
\hline & Gender & 0.139 & 0.001 & 0.157 & $<0.001$ \\
\hline & Height $(\mathrm{cm})$ & -0.035 & 0.412 & - & - \\
\hline & Weight (kg) & -0.014 & 0.744 & - & - \\
\hline & BMI $\left(\mathrm{kg} / \mathrm{m}^{2}\right)$ & 0.050 & 0.242 & - & - \\
\hline & $\mathrm{SBP}(\mathrm{mmHg})$ & -0.055 & 0.197 & - & - \\
\hline & $\mathrm{DBP}(\mathrm{mmHg})$ & 0.020 & 0.644 & - & - \\
\hline & Heart ratio & -0.033 & 0.433 & - & - \\
\hline & Sleep duration per day (min) & 0.010 & 0.814 & - & - \\
\hline & IOP $(\mathrm{mmHg})$ & 0.057 & 0.182 & - & - \\
\hline & Axial length (mm) & -0.080 & 0.058 & - & - \\
\hline & Overall SCP PD (\%) & -0.250 & $<0.001$ & -0.264 & $<0.001$ \\
\hline & $\mathrm{FAZ}\left(\mathrm{mm}^{2}\right)$ & -0.067 & 0.115 & - & - \\
\hline & Overall DCP (\%) & -0.023 & 0.582 & - & - \\
\hline \multirow[t]{14}{*}{$\mathrm{FAZ}\left(\mathrm{mm}^{2}\right)$} & Age (yrs) & 0.034 & 0.423 & 0.053 & 0.207 \\
\hline & Gender & 0.169 & $<0.001$ & 0.156 & $<0.001$ \\
\hline & Height (cm) & -0.023 & 0.584 & - & - \\
\hline & Weight (kg) & -0.082 & 0.052 & - & - \\
\hline & BMI $\left(\mathrm{kg} / \mathrm{m}^{2}\right)$ & -0.038 & 0.375 & - & - \\
\hline & $\mathrm{SBP}(\mathrm{mmHg})$ & 0.032 & 0.449 & - & - \\
\hline & $\mathrm{DBP}(\mathrm{mmHg})$ & -0.039 & 0.355 & - & - \\
\hline & Heart ratio & -0.039 & 0.363 & - & - \\
\hline & Sleep duration per day (min) & 0.013 & 0.753 & - & - \\
\hline & IOP $(\mathrm{mmHg})$ & -0.056 & 0.184 & - & - \\
\hline & Axial length (mm) & -0.135 & 0.001 & -0.123 & 0.004 \\
\hline & Overall SCP PD (\%) & -0.015 & 0.723 & - & - \\
\hline & Overall DCP PD (\%) & -0.067 & 0.115 & - & - \\
\hline & Overall PVD (\%) & -0.032 & 0.450 & - & - \\
\hline \multirow[t]{7}{*}{ Overall PVD (\%) } & Age (yrs) & -0.058 & 0.168 & -0.020 & 0.630 \\
\hline & Gender & 0.126 & 0.003 & 0.089 & 0.029 \\
\hline & Height $(\mathrm{cm})$ & -0.041 & 0.331 & - & - \\
\hline & Weight (kg) & -0.047 & 0.268 & - & - \\
\hline & BMI $\left(\mathrm{kg} / \mathrm{m}^{2}\right)$ & -0.006 & 0.896 & - & - \\
\hline & $\mathrm{SBP}(\mathrm{mmHg})$ & -0.005 & 0.906 & - & - \\
\hline & $\mathrm{DBP}(\mathrm{mmHg})$ & -0.002 & 0.959 & - & - \\
\hline
\end{tabular}

(Continued) 
Table 3 (Continued).

\begin{tabular}{|l|l|l|l|l|l|}
\hline & Variables & Unadjusted Coefficients $(\boldsymbol{\beta})$ & $\boldsymbol{P}$ & Adjusted Coefficients $(\boldsymbol{\beta})^{*}$ & $\boldsymbol{P}$ \\
\hline & Heart ratio & 0.056 & 0.188 & - & - \\
& Sleep duration per day (min) & 0.024 & 0.564 & - & - \\
& IOP (mmHg) & -0.057 & 0.180 & - & - \\
& Axial length (mm) & -0.183 & $<0.001$ & -0.158 & - \\
& Overall SCP PD (\%) & 0.266 & $<0.001$ & 0.251 & $<0.001$ \\
& Overall DCP PD (\%) & -0.023 & 0.582 & - & - \\
& FAZ $\left(\mathrm{mm}^{2}\right)$ & -0.032 & 0.450 & - & - \\
\hline
\end{tabular}

Note: *Adjusted with significant factors by univariate regression analysis.

Abbreviations: BMI, body mass index; DBP, diastolic blood pressure; DCP, deep capillary plexus; FAZ, foveal avascular zone; IOP, intraocular pressure; PVD, peripapillary vessel density; SBP, systolic blood pressure; SCP, superficial capillary plexus.

findings. Otherwise, in the Hsu et al study, the superficial vascular complex (SVC) was predicted by age, and the relationship between age and SVC was quadratic. In addition, Leng et al studied individuals aged $8-87$ years and found decreasing vessel density with increasing age. ${ }^{19}$ Interestingly, the macular vessel density and PVD were steady for adolescents aged 11-18 years. However, their results cannot be directly compared with those of our study due to differences in race/ethnicity, devices used, and software employed.

In our study, both macular vascular density and optic disc vascular density were associated with sex, and girls have a larger inferior hemiretinal SCP-PD, DCP-PD, and FAZ than boys. Our findings were in accordance with those of previous studies. ${ }^{19}$ Previous analyses have concluded from OCT evaluations that girls have thinner retinas than boys, ${ }^{20,21}$ which may explain why girls have a larger FAZ area. However, in our study, results suggest that the other locations of SCP-PD had no statistically significant differences, whereas the PVD showed significant differences between the sexes. On the contrary, Zhang et al reported on 71 normal eyes from 71 subjects (mean \pm age, $127.19 \pm$ 4.61 months; range, 53-243 months) and found significant differences in the SCP-PD between boys and girls, but no statistically significant difference in the PVD. ${ }^{20}$ In Borrelli et al study, male sex was associated with increased SCP$\mathrm{PD},{ }^{16}$ which is consistent with our findings. These variations with our study may be explained by differences in study design, age range, or the number of samples per age group.

For the association between retinal vessel density and systematic characteristics, our study findings suggest that $\mathrm{AL}$ was significantly correlated with the FAZ area and PVD. This result is in line with Cheung et al study of children aged 6 to 8 years which reported that an enlarged
FAZ area was associated with decreased AL. ${ }^{23}$ This may be explained by the increased efficiency of the circulation or retinal blood flow in eyes with a shorter $\mathrm{AL}^{23,24}$ However, these findings are different compared with another study that reported AL was only associated with the inside-disc RPC density rather than the FAZ area. ${ }^{19}$ Taken together, the current knowledge regarding the relationship between retinal microvasculature and other ocular characteristics is still sparse.

Interestingly, we further examined the associations between demographic, systematic, and ocular factors using OCTA metrics. In the current study, results suggest that there were no significant relationships between OCTA metrics and age, sleep duration per day, height, BMI, and blood pressure. Inconsistent with our findings, Cheung et al investigated children from Hong Kong aged 6-8 years and found that a larger BMI was associated with decreased retinal vessel density. ${ }^{22}$ A previous crosssectional study involving 52 healthy pediatric patients with mean age $\pm \mathrm{SD}$ of $11 \pm \mathrm{X}$ years showed that a larger FAZ area was significantly associated with older age, ${ }^{15}$ which is inconsistent with our findings. Additionally, a study from Taiwan, Japan, included 145 normal subjects (mean \pm SD age, $53.3 \pm 14.6$ years) and showed that age independently affected the DCP-PD and FAZ in Japanese adults, ${ }^{25}$ whereas in our study, DCP-PD was not likely to be affected by systemic or ocular parameters. This suggests that we can use these OCTA metrics to observe microvascular abnormalities in northern Chinese adolescents. In addition, in a previous prospective cross-sectional study in France, the SCP density was associated with the cardiovascular risk profile and with impaired left ventricular ejection fraction (LVEF) in patients with a high-risk cardiovascular status. $^{26}$ Furthermore, another cross-sectional study among French 
suggested that lower inner vessel length density was associated with acute coronary syndrome (ACS). Thus, OCTA seems to be a potential assessment tool to reflect systemic micro-vascularization's status. Further studies are needed to clarify these findings. ${ }^{27}$

\section{Strengths and Limitations}

The strength of the current study was the relatively large school-based sample design. However, there were some limitations in our study. First, we included some participants with low and moderate myopia or hyperopia which may have affected the results. Second, we did not provide mydriasis to participants, which may have resulted in missing abnormalities within the periretinal area. Third, this study examined only the superficial FAZ and not the deep FAZ. This was because the deep FAZ was not as clearly imaged as the superficial FAZ. FAZ value may be different in SCP and DCP, further studies are needed to describe the DCP data. Fourth, it was difficult to acquire good quality images due to poor cooperation in subjects younger than age 11 years; thus, only participants aged 11 to 18 years were included in this study. Fifth, we did not adjust the scan signal strength index (SSI), rather we excluded eyes with poor- or low-quality images. Sixth, the cross-sectional design of the current study limits our ability to draw conclusions regarding the outcome of demographic or systematic characteristics on retinal microvasculature. Finally, our findings were based on Asian adolescents and with a specific device. It cannot be extrapolated to other parts of the world or to other ethnic groups and other OCTA devices. Further longitudinal multi-ethnical study is necessary to assess the long-term implications of these factors on retinal vascular density.

\section{Conclusions}

The results of our cross-sectional study outline the characteristics and factors of macular vessel density and optic disc vessel density of normal children in a northern Chinese county. Our findings suggest that the inferior hemiretinal SCP-PD, DCP-PD, FAZ, and PVD were larger in girls than in boys. Results suggest that the morphology of retinal microvasculature (DCP-PD, FAZ area, and PVD) was influenced by sex rather than age. With regard to other ocular factors, the FAZ area and PVD were negatively correlated with AL. Notably, macular vessel density and optic disc vessel density in OCTA metrics were stable in adolescents aged 11 to 18 years, and sex and AL should be considered when assessing the OCTA metrics in children. This study provided useful information to further the understanding of retinal vascular development and OCTA clinical applications in adolescents.

\section{Acknowledgments}

We thank the staff and participants in The Tuyou County Pediatric Eye Study for their help and support. No one received financial compensation for their contributions.

This article is supported by the National Natural Science Foundation of China (No. 81300783), China Postdoctoral Science Foundation (No. 2019TQ0358; No. 2019M661162), LiaoNing Revitalization Talents Program (No. XLYC1807082); Shenyang Young and Middle-aged Science and Technology Innovation Talent Support Program (RC190146); "Grassland Elite” Project of the Inner Mongolia Autonomous Region (No. 2020094) and Bethune Charitable Foundation (No. BJ-LM2018014J; No. BJ-LM2019008J). The funders had no role in study design, data collection and analysis, decision to publish, or preparation of the manuscript.

\section{Disclosure}

The authors report no conflicts of interest for this work.

\section{References}

1. Hansen MH, Li XQ, Larsen M, et al. Five-year change in choroidal thickness in relation to body development and axial eye elongation: the CCC2000 eye study. Invest Ophthalmol Vis Sci. 2019;60:3930-3936. doi:10.1167/iovs.19-26807

2. Su Z, Ye P, Teng Y, Zhang L, Shu X. Adverse reaction in patients with drug allergy history after simultaneous intravenous fundus fluorescein angiography and indocyanine green angiography. $J$ Ocul Pharmacol Ther. 2012;28:410-413. doi:10.1089/jop.2011.0221

3. Mameli C, Invernizzi A, Bolchini A, et al. Analysis of retinal perfusion in children, adolescents, and young adults with type 1 diabetes using optical coherence tomography angiography. $J$ Diabetes Res. 2019;2019:5410672. doi:10.1155/2019/5410672

4. Bowl W, Bowl M, Schweinfurth S, et al. OCT angiography in young children with a history of retinopathy of prematurity. Ophthalmol Retina. 2018;2:972-978. doi:10.1016/j.oret.2018.02.004

5. Koulisis N, Moysidis SN, Yonekawa Y, et al. Correlating changes in the macular microvasculature and capillary network to peripheral vascular pathologic features in familial exudative vitreoretinopathy. Ophthalmol Retina. 2019;3:597-606. doi:10.1016/j.oret.2019.02.013

6. Battaglia Parodi M, Romano F, Cicinelli MV, et al. Retinal vascular impairment in best vitelliform macular dystrophy assessed by means of optical coherence tomography angiography. Am J Ophthalmol. 2018;187:61-70. doi:10.1016/j.ajo.2017.12.013

7. Rabiolo A, Marchese A, Sacconi R, et al. Refining Coats' disease by ultrawidefield imaging and optical coherence tomography angiography. Graefes Arch Clin Exp Ophthalmol. 2017;255:1881-1890. doi:10.1007/s00417017-3794-7

8. Spaide RF, Fujimoto JG, Waheed NK, Sadda SR, Staurenghi G. Optical coherence tomography angiography. Prog Retin Eye Res. 2018;64:1-55.

9. Lonngi M, Velez FG, Tsui I, et al. Spectral-domain optical coherence tomographic angiography in children with amblyopia. JAMA Ophthalmol. 2017;135:1086-1091. doi:10.1001/jamaophthalmol. 2017.3423 
10. Mansour AM, Stewart MW, Yassine SW, et al. Unmeasurable small size superficial and deep foveal avascular zone in nanophthalmos: the collaborative nanophthalmos OCTA study. $\mathrm{Br} J$ Ophthalmol. 2019;103:1173-1178. doi:10.1136/bjophthalmol-2018-312781

11. Okawa Y, Maruko I, Kawai M, et al. Foveal structure and vasculature in eyes with idiopathic epiretinal membrane. PLoS One. 2019;14: e0214881. doi:10.1371/journal.pone.0214881

12. Golebiewska J, Olechowski A, Wysocka-Mincewicz M, et al. Optical coherence tomography angiography vessel density in children with type 1 diabetes. PLoS One. 2017;12:e0186479. doi:10.1371/journal. pone.0186479

13. Garrity ST, Iafe NA, Phasukkijwatana N, Chen X, Sarraf D. Quantitative analysis of three distinct retinal capillary plexuses in healthy eyes using optical coherence tomography angiography. Invest Ophthalmol Vis Sci. 2017;58:5548-5555. doi:10.1167/iovs.17-22036

14. Ghassemi F, Fadakar K, Bazvand F, Mirshahi R, Mohebbi M, Sabour S. The quantitative measurements of vascular density and flow areas of macula using optical coherence tomography angiography in normal volunteers. Ophthalmic Surg Lasers Imaging Retina. 2017;48:478-486. doi:10.3928/23258160-20170601-06

15. Li S, Yang X, Li M, et al. Developmental changes in retinal microvasculature in children: a quantitative analysis using optical coherence tomography angiography. Am J Ophthalmol. 2020;219:231-239. doi:10.1016/j.ajo.2020.05.008

16. Borrelli E, Lonngi M, Balasubramanian S, et al. Macular microvascular networks in healthy pediatric subjects. Retina. 2019;39:1216-1224. doi:10.1097/IAE.0000000000002123

17. Lu P, Xiao H, Liang C, Xu Y, Ye D, Huang J. Quantitative analysis of microvasculature in macular and peripapillary regions in early primary open-angle glaucoma. Curr Eye Res. 2020;45:629-635. doi:10.1080/02713683.2019.1676912

18. Chu Z, Zhang Q, Zhou H, et al. Quantifying choriocapillaris flow deficits using global and localized thresholding methods: a correlation study. Quant Imaging Med Surg. 2018;8:1102-1112. doi:10.21037/qims.2018.12.09
19. Leng Y, Tam EK, Falavarjani KG, Tsui I. Effect of age and myopia on retinal microvasculature. Ophthalmic Surg Lasers Imaging Retina. 2018;49:925-931. doi:10.3928/23258160-20181203-03

20. Zhang Y, Zhang B, Fan M, et al. The vascular densities of the macula and optic disc in normal eyes from children by optical coherence tomography angiography. Graefes Arch Clin Exp Ophthalmol. 2020;258:437-444. doi:10.1007/s00417-019-04466-0

21. Huynh SC, Wang XY, Rochtchina E, Mitchell P. Distribution of macular thickness by optical coherence tomography: findings from a population-based study of 6-year-old children. Invest Ophthalmol Vis Sci. 2006;47:2351-2357. doi:10.1167/iovs.05-1396

22. Zhang Z, He X, Zhu J, Jiang K, Zheng W, Ke B. Macular measurements using optical coherence tomography in healthy Chinese school age children. Invest Ophthalmol Vis Sci. 2011;52:6377-6383. doi:10.1167/iovs.11-7477

23. Cheung CY, Li J, Yuan N, et al. Quantitative retinal microvasculature in children using swept-source optical coherence tomography: the Hong Kong Children Eye Study. Br J Ophthalmol. 2018.

24. Shimada N, Ohno-Matsui K, Harino S, et al. Reduction of retinal blood flow in high myopia. Graefes Arch Clin Exp Ophthalmol. 2004;242:284-288. doi:10.1007/s00417-003-0836-0

25. Benavente-Perez A, Hosking SL, Logan NS, Broadway DC. Ocular blood flow measurements in healthy human myopic eyes. Graefes Arch Clin Exp Ophthalmol. 2010;248:2287. doi:10.1007/s00417-0101407-9

26. Sato R, Kunikata H, Asano T, et al. Quantitative analysis of the macula with optical coherence tomography angiography in normal Japanese subjects: the Taiwan Study. Sci Rep. 2019;9:8875. doi:10.1038/s41598-019-45336-3

27. Arnould L, Guenancia C, Azemar A, et al. The EYE-MI pilot study: a prospective acute coronary syndrome cohort evaluated with retinal optical coherence tomography angiography. Invest Ophthalmol Vis Sci. 2018;59:4299-4306. doi:10.1167/iovs.18-24090
International Journal of General Medicine

\section{Publish your work in this journal}

The International Journal of General Medicine is an international, peer-reviewed open-access journal that focuses on general and internal medicine, pathogenesis, epidemiology, diagnosis, monitoring and treatment protocols. The journal is characterized by the rapid reporting of reviews, original research and clinical studies across all disease areas. The manuscript management system is completely online and includes a very quick and fair peer-review system, which is all easy to use. Visit http://www.dovepress.com/ testimonials.php to read real quotes from published authors. 